\title{
Significados de las prácticas de cuidado realizadas por cuidadores formales de personas con trastorno de conducta en Cundinamarca (Colombia)
}

\author{
Meanings of care practices performed by formal caregivers of people \\ with conduct disorder in Cundinamarca (Colombia)
}

\section{Resumen}

El artículo presenta una investigación de enfoque interpretativo mediante estudio de caso, entrevistas semiestructuradas y observación participante que buscó comprender las significaciones de las prácticas de cuidado de cuidadores formales de niños, adolescentes y adultos con trastorno de la conducta o trastorno mental psicosocial en un programa de protección en Cundinamarca, Colombia. Se interpretaron prácticas relacionadas con hábitos, salud, formación y educación. Los cuidadores realizan su labor con compromiso, amor, vocación y preocupación por el otro, no obstante, el tiempo de trabajo, actividades de atención y características de la población generan problemas de salud física, estrés, agotamiento, síndrome de burnout y menos interacciones familiares y sociales. Se concluye que para la atención de personas con problemas en su desarrollo o salud se requiere implementar espacios para el cuidado del cuidador, racionalización de actividades y mejor soporte institucional que garantice condiciones para el bienestar ${ }^{1}$ del cuidador y la persona cuidada.

\section{Palabras clave}

Cuidado, prácticas de cuidado, relaciones de cuidado, ética del cuidado, perfil del cuidador.

\begin{abstract}
The article presents an interpretative approach research through case study, semi-structured interviews and participant observation that sought to understand the meanings of formal caregivers' care practices of children, adolescents and adults with conduct disorder or psychosocial mental disorder in a program of protection in Cundinamarca, Colombia. Practices related to habits, health, training and education were interpreted. The caregivers perform their work with commitment, love, vocation and concern for the other, however working time, attention activities and characteristics of the population generate physical health problems, stress, exhaustion, burnout syndrome and less family and social interactions. It is concluded that for the attention of people with problems in their development or health, it is necessary to implement spaces for the care of the caregiver, rationalization of activities and better institutional support that guarantees conditions for the welfare of the caregiver and the person cared for.
\end{abstract}

\section{Keywords}

Care, care practice, relationship of care, ethics of care, caregiver profile.

\footnotetext{
1. Se aborda desde el planteamiento realizado por Mulsow (2008) quien refiere que el bien-estar hace parte del proceso de ampliación de las opciones de las personas en su desarrollo cuente con los recursos necesarios para mantener el nivel de vida decente que favorezca su bien-ser, bien-estar, bien-hacer y bien-saber. El bien-estar comprende al individuo como ser que se siente bien dentro de su existencia.
}

\author{
Lady Lorena Hormaza \\ <lololorito2@gmail.com>
}

Universidad Pedagógica Nacional. Colombia

\section{Franci Camila Amezquita \\ Torres}

<camilatorres.0311@gmail.com>

Universidad Pedagógica Nacional. Colombia

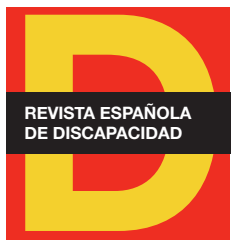

Para citar:

Hormaza, L. L. y Amezquita, F. C. (2018): "Significados de las prácticas de cuidado realizadas por cuidadores formales de personas con trastorno de conducta en Cundinamarca (Colombia)". Revista Española de Discapacidad, 6 (I):139-159

Doi: <https://doi.org/10.5569/23405104.06.01.07>

Fecha de recepción: 12-10-2017 Fecha de aceptación: 20-02-2018

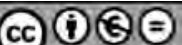




\section{Introducción}

Las prácticas de cuidado están orientadas a distintas poblaciones que, por sus características, necesitan de un apoyo externo como la discapacidad o trastornos destructivos del control de los impulsos y de la conducta; según el "Manual diagnóstico y estadístico de los trastornos mentales DMS-5" (American Psychiatric Association, 2014a) dichos trastornos están conformados por un conjunto de comportamientos que denotan déficit en el control de impulsos; entre ellos se encuentra el trastorno negativista desafiante, el trastorno explosivo, el trastorno de la conducta, el trastorno de la personalidad antisocial, la piromanía, la cleptomanía, y otros trastornos destructivos del control de los impulsos y de la conducta tanto especificados como no especificados. En este sentido, la Clasificación Internacional de Enfermedades CIE 10 (Organización Mundial de la Salud, 2001) presenta al trastorno oposicionista desafiante como un diagnóstico de los trastornos de la conducta caracterizado por un patrón repetitivo de comportamiento asocial y agresivo donde se evidencia la constante transgresión a la norma; siendo estas las características que los diferencian de otro tipo de trastornos.

Ahora bien, el trastorno mental psicosocial es una categoría que no ha sido contemplada desde documentación guía manejada por áreas como la psiquiatría o la psicología; esta ha sido definida por el Instituto Colombiano de Bienestar Familiar (ICBF) (2006) para clasificar a poblaciones que presentan alteraciones de la conciencia, impulsos, problemas de atención y de control del temperamento vistos como problemáticos en los contextos sociales donde interactúan. Por tanto, requieren de apoyo terapéutico desde distintas áreas profesionales y su vinculación a espacios sociales, educativos y culturales donde se busca que obtengan aprendizajes necesarios para su adecuado desenvolvimiento personal y social (Instituto Colombiano de Bienestar Familiar, 2006).

El cuidado en este tipo de diagnósticos está enmarcado en el diseño e implementación de estrategias que conlleven al establecimiento e implementación de normas en aras de que permitan que el niño, niña, adolescente o adulto reconozca las figuras de autoridad y se disminuyan los comportamientos disruptivos, el descontrol de impulsos y alteraciones comportamentales; de igual manera, en algunas ocasiones se hace uso de fármacos para la regulación de la conducta.

Muchos padres asumen el cuidado de su hijo con responsabilidad y compromiso como cuidadores artesanales (De la Cuesta, 2004), brindan apoyo complementario a los tratamientos médicos, acompañan los procesos psicológicos (Granadillo, 2010) y educativos (Noddings, 2005) que reciben. Así pues, entidades o programas resaltan la importancia de la familia como entorno protector (UNICEF, 2006), no obstante, la presencia de un niño con alguno de los diagnósticos mencionados y sin el acompañamiento terapéutico que requiere puede causar tensiones en la familia y dificultades en la convivencia llevando a que reciba malos tratos o no cuente con pautas de crianza claras y desarrolle conductas oposicionistas desafiantes (Zingraff et al., 1993) lo que conlleva a que la familia busque alternativas como internarlo en una institución o incluso el abandono (Santamaría, 2013).

Colombia, en coherencia con la Convención sobre los Derechos del Niño (UNICEF, 2006) y la Constitución Política de 1991 (Corte Constitucional, 2015) cuenta con el Instituto Colombiano de Bienestar Familiar y la Secretaría de Integración Social que, como instituciones del Estado, tienen la responsabilidad de la aten- 
ción, el cuidado y la protección e integración familiar y social de niños, niñas, adolescentes y algunos adultos que por su condición de vulnerabilidad física, psicológica y social, deben seguir bajo protección, a través de programas integrales de calidad con enfoque intersectorial, territorial y diferencial de atención, protección o rehabilitación para que posibiliten oportunamente el restablecimiento de derechos o la reintegración a la sociedad. Esta atención se puede brindar en instituciones bajo la modalidad de internado o a través de la integración de los niños en el sistema escolar, dependiendo del trastorno que presentan y si sus derechos se encuentran inobservados, amenazados o vulnerados (Instituto Colombiano de Bienestar Familiar, 2006).

Con la promulgación del Código de la Infancia y Adolescencia² se asumió la defensa de niños, niñas, adolescentes y aquellos adultos que estén en protección del ICBF a través del ejercicio pleno de los derechos de los cuales son sujeto, la prevención de su vulneración y restablecimiento inmediato.

Lo anterior concuerda con lo expuesto por la Organización Mundial de la Salud (OMS, 2004) en cuanto a que los niños, niñas, adolescentes y adultos con problemas en su desarrollo tienen derecho a disfrutar plenamente en condiciones de igualdad y equidad de todos los derechos y libertades fundamentales como el acceso a la educación, los servicios de salud, recreación y garantizar el reconocimiento y respeto de su dignidad a través del cuidado integral.

Diferentes investigaciones se han centrado en el rol del cuidador y las afectaciones que puede padecer en la atención de personas con algún tipo de diagnóstico como el alzhéimer (Cerquera y Galvis, 2014); una de las consecuencias es la pérdida progresiva de energía hasta el agotamiento, síntomas de ansiedad, depresión, desmotivación en su trabajo y agresividad lo que ha llevado a abordar el tema de cuidado en relación al síndrome de burnout trabajado desde 1974 por el psiquiatra Herbert Freudenberger quien explicó que éste consiste en fallar, desgastarse o sentirse exhausto debido a las demandas excesivas de energía, fuerza o recursos (Gutiérrez Aceves et al., 2006: 306). En relación a este tema, se encuentran investigaciones como las de Torres (2010) en las que muestra que la sobrecarga por el cuidado de personas de tercera edad genera deterioro en los ámbitos laborales, personales e interpersonales del cuidador, por lo que menciona la importancia de generar espacios orientados a mejorar estos aspectos. Otras investigaciones se enfocan en las actividades, el tiempo y la calidad de atención de cuidado que se brinda, especialmente a los niños y niñas (Berlin, 2005; Berlin et al., 2008).

El rol de la mujer en el cuidado también ha sido objeto de estudio, pues históricamente se les ha delegado esta labor dejando de lado su autocuidado debido a que las necesidades de quien cuida se vuelven más relevantes que las propias (Milosavljevic, 2007); además, se afirma que el rol de mujer cuidadora en el sector formal es más representativo que en el informal porque se relaciona directamente con la profesión de enfermería (Espinoza et al., 2011) y en el segundo porque deciden llevar a cabo esta práctica sin tener un parentesco con quien es cuidado (Villarejo et al., 2012).

Cabe decir que el cuidador, sea formal o informal, se ha relacionado con la dependencia parcial o total del paciente a quien se le brinda el acompañamiento y cuidado; esto genera que ambos se vean afectados ya que pueden recibir malos tratos verbales o físicos en la relación de cuidado (Carretero et al., 2006) como consecuencia de las condiciones y jornadas de cuidado.

2. Colombia. Ley 1098 de 2006 por la cual se expide el Código de la Infancia y la Adolescencia. Diario Oficial, 8 de noviembre de 2006 , núm. 46.446 . 
Las investigaciones de Meana (2009), Hernández y Ehrenzweigs (2008) se centran en la caracterización del cuidador y en medir la sobrecarga pero no se evidencia su percepción frente a la práctica que realizan, pues los resultados que arrojan estas investigaciones son de tipo descriptivo de las variables sociodemográficas y de la carga en el cuidador.

Es de mencionar que para la elaboración de los antecedentes empíricos no se encontraron estudios relacionados con el cuidado de niños, niñas, adolescentes y adultos en instituciones de protección que presenten un diagnóstico de trastorno de la conducta o trastorno mental psicosocial, de ahí la pertinencia de la investigación y la importancia de comprender la concepción de las personas encargadas del cuidado de esta población, pues se entiende que el cuidado va siempre acompañado de una serie de prácticas individuales y colectivas que le dan sentido las cuales junto con las expectativas y los requerimientos que reviste, permiten o no brindar atención especializada.

\section{Referente teórico}

Teniendo en cuenta que con la investigación se buscó interpretar las significaciones de las prácticas de cuidado en los cuidadores formales que trabajan en una institución de protección, se parte de la elaboración del soporte teórico tomando cuatro perspectivas para el abordaje de las prácticas de cuidado: la de Michael Foucault (1987), quien plantea el cuidado de sí mismo; Martha Nussbaum (2012), quien las pone en el contexto de la familia; Carmen de la Cuesta (2004), quien las propone desde la práctica en enfermería y en un contexto informal planteándolo como un trabajo artesanal; y, por último, Thomas Luckmann (2008) quien las orienta hacia la construcción del otro desde el cuidado mediante la interacción social que los sujetos realizan en el mundo de la vida durante los procesos de socialización.

Existen otras posturas que aportan al concepto de cuidado como la de Carol Gilligan (1982) quien plantea la relación de cuidado como un diálogo a través de la preocupación del otro concreto y el reconocimiento de sus necesidades, además hace una crítica al juicio moral expuesto por Kohlberg (1981) afirmando que el cuidado es una labor que no sólo compromete a la mujer. De igual modo, sustenta la importancia de comprender las relaciones de cuidado entre los cuidadores y la población, donde no sólo los primeros sean los que deben llevar a cabo el cuidado sino también quienes lo reciben, con el fin de que sea una relación recíproca desde la comprensión de las necesidades del otro (Noddings, 2005).

Los seres humanos siempre se han preocupado por la protección de la vida creando prácticas, principios y normas para garantizarla; el cuidado inevitablemente remite a la existencia humana y emerge de su subjetividad; también se configura como prácticas artesanales (De la Cuesta, 2004) y profesionales (Hernández y Ehrenzweigs, 2008), como una manera de estar ahí, con conciencia, disposición, con autoconocimiento y como cuidador o cuidadora de otra u otras personas que están en el mundo de la vida, en ocasiones, bajo circunstancias especiales. 
Siguiendo lo anterior, se retoma la afirmación de Heidegger quien menciona que "El cuidado como forma de ser esencial es algo más que un acto y una actitud entre otras" (1951: 33). Desde esta perspectiva, el cuidado, en alemán Cure, cura o Sorge, significa "cuidar de» y «velar por», remite al cuidado de otros y al cuidado de cosas e implica un hacer con un fin previsto a través de la praxis; es también ocuparse de «sí mismo" (Murueta, 2009). Es una actitud en cuanto a disposición, responsabilidad, compromiso, ocupación e involucramiento (Boff, 2002). Heidegger dice en su obra "Ser y Tiempo": "Desde el punto de vista existencial, el cuidado se encuentra a priori, antes de toda actitud y situación del ser humano, lo que significa decir que el cuidado está presente en toda actitud y situación de hecho" (1976: 33); de ahí que el cuidado sea una dimensión fundacional de la vida humana, es inherente a su naturaleza y condición, a través de él se revela cómo es el ser humano.

Desde la perspectiva teológica de Leonardo Boff (2002) el cuidado requiere de dos actitudes; el desvelo y la atención hacia quien se cuida, y la preocupación por garantizar su reconocimiento en la relación de cuidado. El ejercicio de cuidar presenta un contenido axiológico donde se desencadenan cuestiones afectivas y morales que conllevan a que los cuidadores refieran que su labor se ejerce con amor (De la Cuesta, 2004). Pero el cuidado, además de relacionarse con acciones que beneficien a otras personas, también se encuentra en la manera en que los seres humanos se cuidan a sí mismos, tanto física como emocionalmente (Foucault, 1987).

El cuidado se ejerce por medio de prácticas conformadas por conductas de protección a la salud (Harris y Guten, 1979), definidas en acciones materializadas cuando el cuidador crea y desarrolla estrategias que den respuesta a las necesidades y el bienestar (Mulsow, 2008) de quien cuida, las cuales se instauran y son reproducidas. Thomas Luckmann (2008) explica las relaciones de los actores desde un proceso que emerge en la acción meramente dicha o desde el pensamiento; aquí las acciones sociales están proyectadas hacia "el otro" donde se le incluye como actor principal o espectador. Tomando como partida esta premisa, se afirma que los cuidadores que participaron en la investigación desarrollan acciones que responden a necesidades básicas de la población atendida como en el acompañamiento terapéutico y formación que sea requerida y en las cuales se hace visible la preocupación, dedicación y compromiso (De la Cuesta, 2004).

Para la interpretación de las significaciones que los cuidadores hacen de sus prácticas, se consideró necesario abordar el planteamiento teórico en relación a la definición del cuidador encontrando que es "aquella persona que asiste o cuida a otra afectada de cualquier tipo de discapacidad, minusvalía o incapacidad que le dificulta o impide el desarrollo normal de sus actividades o sus relaciones sociales" (Secretaría de Integración Social y Universidad Nacional de Colombia, 2008: 8); por lo tanto, su labor se orienta a responder a las necesidades físicas, emocionales y económicas que presenta la persona cuidada.

El rol de cuidador comprende el conjunto de prácticas orientadas a la atención de quien no puede responder a todas sus necesidades toda vez que presenta algún tipo de discapacidad, enfermedad o dificultad que perjudica o impide el normal desarrollo de sus actividades vitales, laborales o en la vida social (Flores et al., 1997). Los cuidadores varían en los tipos de cuidado que deben brindar, dependiendo de si existe o no un vínculo familiar con la persona que recibe el cuidado; siendo así, se reconocen dos categorías de cuidadores: formales, quienes tienen un conocimiento técnico del cuidado y reciben una remuneración económica (De la Cuesta, 2004); e informales, quienes desarrollan la labor de cuidado de un familiar, amigo o conocido sin esta remuneración (Aguas, 1999; De la Cuesta, 2004). 
En este mismo sentido Valderrama (1990), tomando el planteamiento expuesto por De los Reyes (2001: 4), indica que los cuidadores formales son "personas capacitadas a través de cursos teóricos-prácticos de formación dictados por equipos multidisciplinarios e interdisciplinarios de docencia para brindar atención preventiva asistencial y educativa a quien se cuida y a su núcleo familiar". Cabe resaltar que este es un concepto aproximado a la labor realizada por los cuidadores de la institución donde se llevó a cabo la investigación teniendo en cuenta que se desarrollan procesos de atenciones asistenciales pero también educativas y terapéuticas en las que se implementan los conocimientos técnicos de los cuidadores, no obstante, las situaciones emergentes que surgen debido a alteraciones comportamentales propias de las características de los diagnósticos que presenta la población conlleva que los cuidadores deban generar estrategias de atención que permitan realizar su labor, pues en muchas ocasiones la realidad exige que los cuidadores trasciendan de sus conocimientos teóricos en aras de responder a los objetivos institucionales.

Por otro lado, las relaciones de cuidado son las dinámicas establecidas entre el cuidador y quien es cuidado, donde se da importancia a las maneras en que el cuidador desarrolla su labor y cómo quien es cuidado entiende los sentimientos y deseos de quien lo atiende, orientando una actitud positiva desde sus capacidades y valores (Noddings, 2005), permitiéndose de esta manera una ética de cuidado a partir del reconocimiento de las necesidades del otro visualizándolo como sujeto, como un otro concreto y un otro generalizado (Gilligan, 1982), surge cuando la existencia de alguien tiene importancia para otro, es decir para quien lo cuida, considerándose como una actitud desde la que se reconoce la existencia y las necesidades del otro (Boff, 2002).

Teniendo en cuenta que la investigación estuvo inmersa en los procesos de cuidado formal y significación de los cuidadores sobre su labor, el referente conceptual que permitió un acercamiento a la interpretación de las prácticas de cuidado fue Jean Watson (1979) tomando como referencia los planteamientos de BáezHernández et al. (2009) quienes lo conciben como una manifestación en la práctica interpersonal que tiene como finalidad promover la salud y el crecimiento de la persona, respondiendo a las necesidades mediante acciones definidas a partir del tipo de ayuda que sea indispensable ya sea extenso, intermitente o limitado y llevar a cabo una práctica del cuidado exitosa. Su teoría propone cinco procesos básicos: conocimiento, estar con, hacer por, posibilitar y mantener las creencias, en los que se hace evidente el cuidado de enfermería que se caracteriza por "la actitud filosófica de la enfermera, la comprensión, los mensajes verbales y no verbales, las acciones terapéuticas y las consecuencias de los cuidados (Báez-Hernández et al., 2009: 129).

\section{Metodología}

\section{- Enfoque y método de investigación}

La investigación se ubicó en el enfoque epistemológico de las ciencias interpretativas, mediante el método de estudio de caso desde la perspectiva de Yin (1984), quien considera que este método es una estrategia de investigación aplicada en un contexto real cuando los límites entre este y el fenómeno no son evidentes; y Stake (1998), quien indica que desde este método se espera abarcar la complejidad de un caso el cual puede estar conformado por un individuo o un grupo con características similares donde la concentración está en abordar a los individuos que conforma el colectivo. 
Teniendo en cuenta lo anterior, se usó el método de estudio de caso instrumental (Stake, 1998) el cual cumple con el rol de mediación para la comprensión de un fenómeno que lo trasciende. Es un instrumento utilizado para evidenciar las características de algún fenómeno; siendo así, en esta investigación se utilizó para interpretar el significado que le dan los cuidadores de una institución de protección a sus prácticas de cuidado ejercidas en población con trastorno de la conducta o trastorno mental psicosocial, sin pretensiones de generalización de los resultados, pero sí con la posibilidad de transferibilidad a casos similares (Pourtois y Desmet, 1992) de modo que se logró un acercamiento a la experiencia, percepción y concepción de los cuidadores.

\section{- Participantes}

Se contó con la participación voluntaria de 9 cuidadores que trabajan en la institución y a quienes se protegió su identidad dejando en anonimato y asignándoles un número como referencia para la presentación de fragmentos de las entrevistas. La selección de los participantes se hizo bajo los criterios de disponibilidad de tiempo para desarrollar la entrevista de manera individual y se buscó abarcar diferentes áreas laborales de la institución para lograr tener la percepción que cada cuidador tiene en cada uno de los cargos que ejerce; además, se conforma un grupo heterogéneo según sexo, estado civil, edad y lugar de residencia con el fin de tener un acercamiento a los cuidadores desde diferentes características.

\begin{tabular}{|c|c|}
\hline Cuidador & Perfil \\
\hline Cuidador 1 & Mujer de 33 años, soltera sin hijos, residente en la ciudad de Bogotá y formadora en la institución. \\
\hline Cuidador 2 & $\begin{array}{l}\text { Hombre de } 23 \text { años quien se encuentra en unión libre, tiene un hijo y reside en el municipio donde se } \\
\text { encuentra la institución, donde asume el cargo de formador. }\end{array}$ \\
\hline Cuidador 3 & $\begin{array}{l}\text { Hombre de } 27 \text { años, separado, tiene un hijo y reside en el municipio donde se encuentra la institución, donde } \\
\text { asume el cargo de auxiliar de enfermería. }\end{array}$ \\
\hline Cuidador 4 & $\begin{array}{l}\text { Mujer de } 30 \text { años, soltera sin hijos, reside en un municipio diferente al lugar donde se encuentra la institución } \\
\text { y asume el cargo de tallerista. }\end{array}$ \\
\hline Cuidador 5 & $\begin{array}{l}\text { Mujer de } 33 \text { años quien se encuentra en unión libre, tiene dos hijos, vive en el municipio en el que se } \\
\text { encuentra ubicada la institución y asume el cargo de formadora. }\end{array}$ \\
\hline Cuidador 6 & $\begin{array}{l}\text { Mujer de } 40 \text { años, soltera con un hijo, reside en la ciudad de Bogotá y asume el cardo de formadora en la } \\
\text { institución. }\end{array}$ \\
\hline Cuidador 7 & $\begin{array}{l}\text { Hombre de } 37 \text { años, soltero con dos hijos, reside en la ciudad de Bogotá y asume el cargo de profesional } \\
\text { dentro de la institución. }\end{array}$ \\
\hline Cuidador 8 & $\begin{array}{l}\text { Hombre de } 34 \text { años, quien se encuentra en unión libre, tiene un hijo, reside en la ciudad de Bogotá y asume el } \\
\text { cargo de profesional en la institución. }\end{array}$ \\
\hline Cuidador 9 & $\begin{array}{l}\text { Mujer de } 42 \text { años, casada con dos hijos, reside en el municipio donde se encuentra ubicada la institución y } \\
\text { asume el cargo de profesional. }\end{array}$ \\
\hline
\end{tabular}




\section{- Instrumentos}

Para la recolección de información se utilizaron entrevistas semiestructuradas (Valles, 1998) aplicadas a cuidadores formales de manera individual en espacios locativos de la institución donde se llevó a cabo la investigación y observación participante mediante diarios de campo de las actividades de atención y cuidado que reciben los niños, niñas, adolescentes y adultos los cuales se elaboraron durante un periodo de 21 días.

\section{- Procedimiento}

Para el desarrollo de la investigación se siguieron cuatro etapas:

Etapa 1: Diseño de instrumento y recolección de información. A través de entrevistas de 40 minutos, hechas a los nueve cuidadores participantes donde se abordaron preguntas de tipo demográfico, concepción y percepción del cuidador sobre su labor, cotidianidad del cuidador dentro y fuera de la institución así como las redes sociales con las que cuenta o la limitación para la construcción de estas. Se realizó el registro de actividades y prácticas de cuidado en 21 diarios de campo durante tres días a la semana en horarios de comidas en el espacio del comedor y actividades de aula de tipo artístico, deportivas y de esparcimiento en las que se encontraban formadores, talleristas y el equipo profesional, en las que se realizó el registro de lo observado y los diálogos entre la población y los cuidadores. Cada cuidador fue informado de los objetivos de la investigación y el uso de la información obtenida con fines exclusivamente investigativos y se procedió a la firma de los consentimientos informados (American Psychiatric Association, 2014b). Las entrevistas fueron grabadas para su respectiva transcripción, análisis e interpretación a partir del uso de algunas estrategias de la teoría fundamentada y el análisis de narrativas.

Etapa 2: Transcripción protocolar y codificación de la información según las categorías deductivas. Efectuadas las entrevistas y elaborados los registros de diario de campo según la pregunta y objetivos de la investigación hasta lograr la saturación de las categorías deductivas. A través del microanálisis línea por línea, se categorizó la información obtenida mediante el uso del software Atlas Ti para posteriormente realizar el análisis descriptivo con el propósito de hacer una primera aproximación a los significados que los participantes le dan a sus prácticas de cuidado de los niños, niñas, adolescentes y adultos a su cargo de acuerdo con sus roles, actividades y temporalidades.

Etapa 3: Análisis axial de carácter estructural para establecer las relaciones entre categorías y subcategorías con el fin de dar un sentido totalizante a las narrativas de cuidado relatadas por los participantes desde el compromiso, la dedicación temporal, el conjunto de actividades y las tensiones que componen las prácticas de cuidado. Además, se identificaron algunas categorías emergentes que le dan un sentido más amplio a dichas prácticas.

Etapa 4: Configuración y reconfiguración constante de significados. A partir de este proceso, se hizo la interpretación de las narrativas mediante una trama de sentido teniendo en cuenta actores, contextos, tiempos y acciones en la cotidianidad de la vida de la población atendida dentro de la institución. Para el análisis de las narrativas se emplearon procedimientos de triangulación y convergencia para lograr la credibilidad y la validez de consenso. 


\section{Resultados y Discusión}

Acorde con el enfoque epistemológico y metodológico de la investigación de estudio de caso, el análisis que se presenta de los resultados es de naturaleza hermenéutico-interpretativa. Los datos obtenidos se sometieron a análisis de contenidos orientado por las categorías deductivas, también con las categorías inductivas que surgieron durante el proceso de análisis de la información. La discusión se realizó en cada una de las categorías haciendo uso de los referentes teóricos abordados en la investigación; en las citas textuales de las entrevistas se veló por preservar el anonimato de los cuidadores.

\section{- El cuidado}

El cuidado desde algunos participantes está relacionado con actividades asistenciales y de formación ya que acompañan el proceso de los niños, niñas, adolescentes y adultos en el aprendizaje de habilidades para la vida y formación técnica en el caso de quienes presentan condiciones cognitivas para este tipo de aprendizajes; esto lo hacen independientemente de su profesión dado que todo el equipo de cuidadores está inmerso en velar por la población y su atención.

“...Nosotros recibimos, digamos nos llega un niño de...de otro hogar, entonces nosotros nos encargamos de formarlos, de llevar una secuencia y formarlos" (Entrevista C1).

Los cuidadores entienden la atención y el cuidado como un conjunto de acciones orientadas al bienestar (Mulsow, 2008) de la población que atiende, a la satisfacción de sus necesidades, formación y orientación de hábitos y habilidades para la interacción social y la posibilidad de alguna ocupación laboral en quienes tienen menor dificultad en su desarrollo o para quienes están en la institución por problemas de comportamiento.

"Nosotros los estamos formando a ellos como personas civilizadas, porque ellos vienen de pronto con que "me levanto y yo salgo y ya, me bañé y dejo el reguero, dejo todo que lo recojan las demás personas", la idea no es esa, la idea es que ellos pasen por acá y que ellos salgan corrigiendo todos esos errores que tenían afuera" (Entrevista C2).

La percepción de cuidado de los participantes se relaciona con el rol del cuidador desde una orientación hacia la enseñanza de diferentes hábitos sociales y personales con los cuales se pretende preparar a los beneficiarios para su construcción de proyecto de vida (figura 1); es una perspectiva del cuidado diferente a la asistencial donde se llevan a cabo diversas estrategias como la implementación de horarios para el desarrollo de rutinas en las que se motiva a la población mediante incentivos como salidas recreativas; estas intervenciones se diseñan de manera inter y transdisciplinaria buscando un ejercicio coordinado de formación y cuidado que logre afrontar las tensiones que se pueden presentar en relación a las demandas de la institución y el ejercicio de cuidado que se desarrolla.

En este mismo sentido, para los cuidadores la labor de cuidado significa formar a los niños, niñas, adolescentes y adultos; se trata de un trabajo que vela por restablecer los derechos que en algún momento fueron vulnerados y buscar la autonomía en la población para su propio cuidado; es una labor que comprende el 
escuchar a la población y el respeto a su situación, por lo tanto, responde a la ética del cuidado de Gilligan (1982). Para tal finalidad, los cuidadores consideran importante la vinculación de la población a otros espacios culturales, educativos y sociales en los que pueda disfrutar experiencias que por su condición no han vivido y usarlos también como refuerzos positivos cuando se busca establecer pautas y rutinas para su proceso de formación. Además, consideran que el cuidado se comprende como una actividad que permite la construcción de un vínculo que no es encontrado por la población en sus familias, por lo que es importante brindarles un cuidado que les permita sentir protección, lo que conlleva un cuidado enmarcado en el amor y la comprensión.

\section{Figura 1. Árbol Categorial-categoría cuidado}

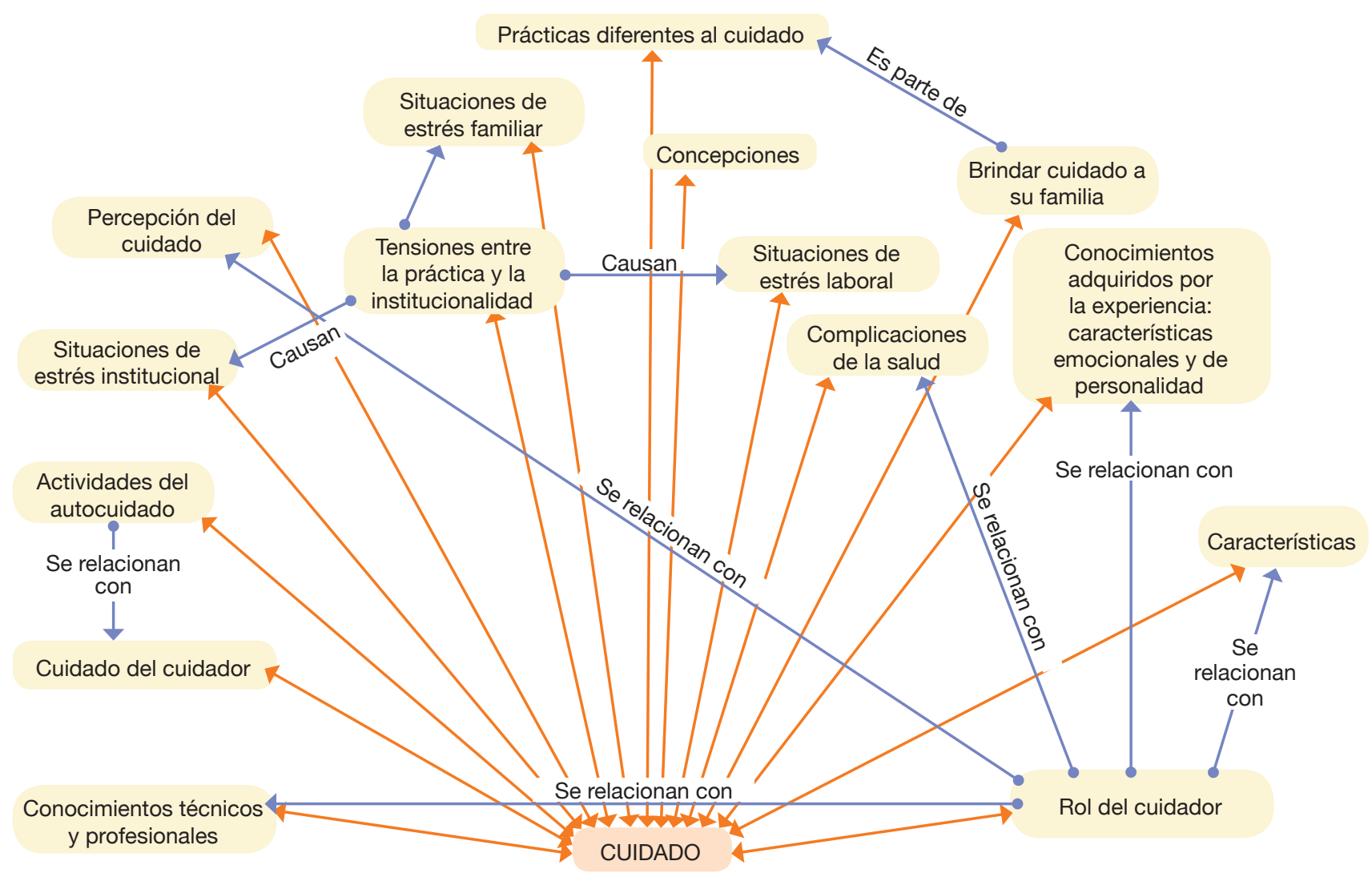

Fuente: elaboración propia a partir de las respuestas dadas por los participantes a las entrevistas.

Los cuidadores entrevistados consideran que el cuidado requiere dedicación con las personas que trabajan; es necesaria la tolerancia, la paciencia, la vocación y la planeación con metas claras que beneficien el desarrollo de la población que atienden. Se requiere carisma para atender a las personas que por sí mismas no se pueden autocuidar. En este aspecto se refleja cómo el cuidado llega a ser un acto de responsabilidad, compromiso que lo convierte en esencial (Boff, 2002). 
“...Uno se plasma metas, y la idea es cumplirlas. Que por lo menos salgan de la visión de que mi grupo es el más fastidioso, los más cansones. Entonces ese es el parecer mío"... "amor por lo que se hace, amor por los chicos, el respeto que se debe también tener eh...frente a los chicos, el compromiso la responsabilidad ¿sí?, la dedicación el...el don ¿no?"... "Eh el amor porque uno sin amor no... o sea uno por hacer las cosas no las debe hacer o sea uno no debe estar trabajando con niños porque le van a pagar a uno, pues obviamente es una necesidad también de la familia o del hogar y todo pero uno lo debe hacer con amor" (Entrevista C2, C4, C6).

Para los funcionarios de la institución la labor de cuidado en poblaciones con algún diagnóstico de la conducta requiere de conocer las pautas de crianza que ha llevado a cabo la familia antes de que el niño, niña, adolescente o adulto llegara a la institución, ya que esto les permite intervenir de manera más asertiva.

El tiempo dedicado a la labor implica para los funcionarios un horario de catorce horas laborales en promedio lo cual conlleva a dejar de lado actividades de interés personal y social perdiendo la posibilidad de disfrutar de ellas, incluso, en ocasiones, el tiempo para compartir con sus amistades y la familia se ve afectado ya sea por el tiempo de trabajo, o por el cansancio físico.

"No hay interacción familiar tampoco hay interacción de amigos, eh...digamos que la vida social se acaba un poco, digamos que ya uno no tiene ese tiempo de "ven vámonos a tomar un café o vamos a irnos a ver una película, o vamos a ir a vernos una obra de teatro" porque los horarios que nosotros manejamos son horarios muy extendidos" (Entrevista C4).

Las condiciones laborales llevan a los participantes a la reorganización de actividades, tiempos y la implementación de prácticas de autocuidado, de manera que su salud física y emocional también sea tenida en cuenta como un aspecto necesario para el cuidado de otros. Consideran que es necesario tener espacios de tiempo para compartir con los compañeros de trabajo, más allá de lo que implica el cumplimiento de sus responsabilidades laborales; en síntesis, es importante fortalecer el cuidado del cuidador, pese a que esta es un área que existe en la institución, no se menciona por las personas entrevistadas, de ahí la necesidad de darle un papel más protagónico en las prácticas de cuidado de sí de los cuidadores.

A partir de los resultados obtenidos en esta categoría se realiza un análisis interpretativo donde se muestra que todas las condiciones de la vida requieren de cuidado como afirma Leonardo Boff (2002), para quien el cuidado se realiza con preocupación y compromiso afectivo con el otro siendo la materialización de un cuidado esencial.

\section{- Prácticas de cuidado}

Dentro de las actividades de atención que deben realizar los cuidadores se encuentran las que están relacionadas con los hábitos de cuidado, regulación y control del comportamiento interpersonal para facilitar la convivencia, actividades de recreación, educativas y de formación, así como también brindar atención a la salud física y psicológica. Todas estas prácticas están orientadas al bienestar (Mulsow, 2008) de la población beneficiaria. Sin embargo, por las características de desarrollo o dificultades comportamentales de algunos de ellos, se pueden presentar manifestaciones de agresividad como golpes e insultos hacia los cuidadores, lo que puede generar estrés laboral y afectar a su salud como es observado a lo largo de los espacios en los que se realizó el registro en diarios de campo. 


\begin{abstract}
"Se solicita por parte de un cuidador a un beneficiario que entregue una gorra debido a que estaba presentando problemas con otro compañero y le estaba golpeando, a lo que el beneficiario responde "ino socio!" y se retira del espacio" (...) "...cuando se indica a la beneficiaria la posibilidad de que participe en la cocina refiere "ive! Por qué si para eso les pagan, para atendernos" a lo cual una cuidadora le aclara que está en un lugar de protección, la beneficiaria se mantiene resistente" (Diarios de campo D6 y D7).
\end{abstract}

Las prácticas de cuidado comprenden múltiples actividades que requieren de estrategias institucionales y de creatividad para garantizar el bienestar (Mulsow, 2008) de los beneficiarios (figura 2), especialmente si se tienen en cuenta las problemáticas que los afectan. Los cuidadores apelan a diferentes mecanismos que estimulen el autocuidado en los usuarios a través de hábitos adecuados. De manera similar emplean estrategias para promover el cumplimiento de las responsabilidades, la convivencia, la adquisición de aprendizajes para la vida y la participación de los usuarios cuyas condiciones físicas y psicológicas lo permiten. Estos procesos de enseñanza requieren de acompañamiento y supervisión permanente. Una tarea importante se relaciona con la convivencia y el afrontamiento de problemas interpersonales y conflictos, en los cuales los cuidadores enseñan la comunicación asertiva, la escucha activa, la mediación en las situaciones de conflicto, el cumplimiento de las normas y negociar cuando las condiciones de la institución o la situación lo permiten. Pese a que la labor del cuidador es la de orientador y solucionador de dificultades, en ocasiones los límites institucionales o del ente gubernamental dificultan esta tarea y las tensiones aumentan perjudicando la relación de cuidado.

Figura 2. Árbol Categorial-categoría práctica de cuidado

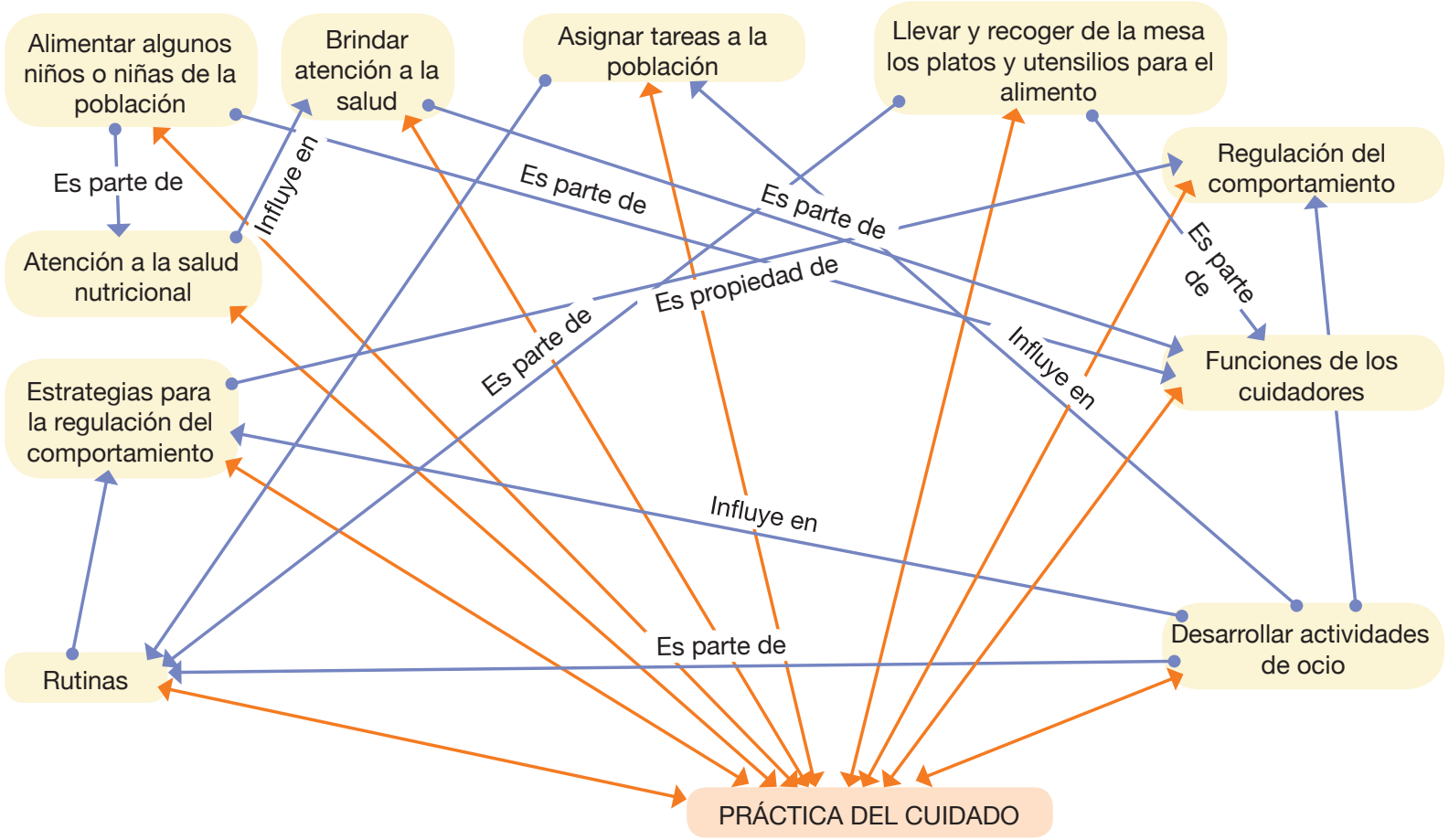

Fuente: elaboración propia a partir de las respuestas dadas por los participantes en las entrevistas. 


\section{- Relaciones de cuidado}

Para los cuidadores, una relación importante que tejen en el desarrollo de su labor diaria es con sus compañeros y con la institución, pues como la mayoría lo menciona, el trabajo del cuidado es un proceso articulado donde se depende del otro para alcanzar los objetivos propuestos, por lo que la falta de comunicación puede generar problemas en el ambiente laboral. Se reconocen como un equipo de trabajo caracterizado por la cooperación y el apoyo; lo anterior no significa que no existan dificultades interpersonales ya sea por baja empatía entre algunos funcionarios, por dificultades en la comunicación, en la percepción o incluso frente a las decisiones que se toman. Algunas de estas dificultades llevan a que algunos cuidadores opten por hacer sus trabajos sin pedir ayuda, como una manera de "sobrevivir" en el trabajo. Más allá de estas dificultades, lo más significativo es que los cuidadores han procurado construir una red de apoyo mutuo. Otra estrategia que han creado a través de la comunicación es la de retroalimentarse en relación con las actitudes en el trabajo, lo que les permite conocer y cambiar comportamientos que dificultan la convivencia y el desarrollo de las actividades.

"Existe la colaboración y de alguna u otra forma, si ya lo hablamos en otros, pues en otros aspectos quizá no es así, porque lo que te digo, acá pues uno hace su trabajo a su manera y es una cuestión también de supervivencia ¿no? O sea, acá el trabajo, no...o sea tu trabajo no lo hace nadie más ¿sí? El trabajo uno responde uno es el que tiene que dar resultados, nadie va hacerlo por ti” (Entrevista C4).

En cuanto a las relaciones entre las directivas y la institución, como en cualquier otra organización, depende de los roles y funciones que tiene a su cargo los cuidadores, no obstante, algunos de ellos consideran que existe un trato diferencial que no reviste los mismos beneficios para todos; en ocasiones estas relaciones percibidas causan malestar e inconformidad, por lo que expresan la necesidad de un trato equitativo.

“...Con las directivas...bien...umm...no, siempre, siempre le he encontrado como respuesta a...a las inquietudes... con...con la otra, bueno en ese si hay muchas cosas que no me parecen...que no se manejan bien...emm...de pronto se ve la diferencia de persona a persona....entonces no me parece cómo se manejan las cosas, aunque no he tenido...encuentros de esos fuertes o...eso, sí hay cosas que realmente molestan y aburren...Por ejemplo lo...que te griten delante de la gente sin ni siquiera escucharte es bastante molesto y bastante...eh...no sé...simplemente porque lo aburre a uno" (Entrevista C3).

El grupo de profesionales de la institución se encuentra organizado a partir de las siguientes áreas: Una parte psico-social para la comunicación constante con la entidad que se encarga de resolver los casos y decidir si se efectúan reintegros al medio familiar; dicha área está compuesta por psicólogos que desarrollan estrategias de intervención terapéutica teniendo en cuenta historia de vida, edad, dinámica familiar y diagnóstico de cada beneficiario; y por trabajadores sociales que velan por el ejercicio de derechos de la población. También se cuenta con otra área compuesta por educadores especiales y terapeutas ocupacionales quienes con apoyo de formadores y talleristas desarrollan actividades de ocio, ocupacionales y educativas. El proceso de atención en cada área varía según características, diagnósticos y edad de la población, así como el nivel educativo ya sea secundario, universitario o de formación para el trabajo. Además, cuenta con otra área compuesta por médicos generales, psiquiatras, nutricionistas y enfermeros, quienes capacitan al talento humano de la entidad en temáticas específicas de acuerdo al perfil de la población, no obstante, los cuidadores entrevistados mencionaron que, a pesar de ser constantemente capacitados, los recursos humanos no son suficientes, especialmente en los fines de semana, ya que las actividades aumentan y se diversifican. 
"En algunos casos el apoyo digamos la parte logística es muy poca, a veces no tenemos las herramientas suficientes para abordar, digamos un fin de semana. Se hace el esfuerzo, se plantea la situación pero a veces no es efectiva la labor" (Entrevista C7).

Bajo lo anterior, se logra afirmar que el cuidado implica una relación en la que se atienda las necesidades de quien es cuidado pero también deben visibilizarse las necesidades del cuidador con el fin de que se encuentre con un espacio comprensivo que le permita desarrollar su labor desde un ambiente tranquilo, pues como menciona Noddings (2005) se deben entender los sentimientos y deseos de quien cuida, lo que ayudaría a prevenir o por lo menos sopesar la sobrecarga del cuidador.

Las relaciones entre los cuidadores y los beneficiarios están mediadas por el diálogo pero es importante resaltar que, en ocasiones, se emplea la palabra como mecanismo de control mediante amenazas y para establecer normas y límites, esto con el fin de que el cuidador deje claro su rol de autoridad ya que como ellos mismos mencionan, al no hacerlo se dificulta la regulación del comportamiento de los beneficiarios.

“...tienes que utilizar a veces la voz fuerte, esa posición corporal donde eh...tienes que darle a conocer al chico que "hey yo soy" eh...entonces pues eh digamos si, de alguna otra manera esa autoridad pues para decir "hey quédate quieto" o "no hagas tal cosa" ¿sí?" (Entrevista C4).

"Se observa que se le llama la atención a una beneficiaria quien se resiste a bajar al aula, por lo tanto se acercan dos profesionales para bajarla pero se tira al piso y grita, frente a esto los dos profesionales la toman de la mano y la bajan, al llegar al aula la beneficiaria nuevamente se tira al piso y dice "perro" a un profesional el cual le dice "¿cómo me está diciendo? Repita, dígalo en la cara" la beneficiaria menciona "ipero mire cómo me dejó!" seguido a esto el profesional le dice "siga de grosera y verá cómo le va" una profesional le dice a la beneficiaria que por favor se quede quieta, pero al no escuchar la solicitud y seguir alterada un profesional refiere que será necesario inmovilizarla cuatro puntos, al referir esto la beneficiaria reacciona y dice gritando "¿por qué?" y una profesional le responde "por grosera" (Diario de campo D19).

Para los cuidadores, su labor debe enmarcarse en el amor y el compromiso con la vida de quien cuida haciendo un reconocimiento de las características propias de cada beneficiario cuidado como ese "otro" en la relación de cuidado (Luckmann, 2008); por lo tanto, se parte por el interés de conocer su historia de vida, pues dentro de la labor de cuidado se construyen vínculos que pueden ser favorables para el cuidador en el momento de establecer normas a la población, así como el reconocimiento de su papel como autoridad.

\section{- Ética del cuidado}

La labor de cuidado realizada en la institución donde se llevó a cabo la investigación busca comprender al otro como un ser humano que tiene diferentes problemáticas y formas distintas de ver y estar en el mundo; esto es relevante ya que, aunque parezca lógico, entender los diagnósticos de los beneficiarios es importante para los cuidadores porque les permite atender mejor a la población y afrontar el estrés que se les genera.

Dentro de los mecanismos de afrontamiento y las actitudes que los cuidadores consideran deben tener mencionan el desarrollo de las prácticas de cuidado con amor, con disposición; aunque, en ocasiones, se pueden presentar abusos de poder, tratos inadecuados o amenazas para que quien es interpelado de esta forma haga las labores que se le asignan. Enfatizan en que es necesario desempeñar sus funciones 
brindando lo mejor a los beneficiarios en todo momento, mediante un trato digno. Por lo cual, la mayoría de los cuidadores procuran realizar sus prácticas de cuidado con compromiso, respeto y responsabilidad en aras de promover el restablecimiento de los derechos de la población y no como una obligación meramente contractual; esto refleja el desarrollo de un cuidado esencial desde la perspectiva de Boff (2002) pero que puede tener tensiones las cuales conllevan a ejercer un cuidado en la adversidad (De la Cuesta, 2004).

“...porque nosotros trabajamos con personas, si nosotros trabajáramos con objetos, los objetos no leen y no interpretan nuestras emociones es todavía más sencillo, pero nosotros trabajamos con personas, ellos también leen cómo nos comportamos, cómo manejamos nosotros las emociones, entonces para nosotros deber ser muy importante manejar las emociones que se puedan generar dentro del trabajo..." (Entrevista C8).

Los resultados reflejan que los funcionarios manifiestan que el cuidado es comprender el mundo de quien se cuida mediante el conocimiento de su diagnóstico, su historia de vida, así como sus necesidades particulares, lo que se relaciona con la ética del cuidado (Gilligan, 1982). En este sentido, la empatía es uno de los componentes clave para establecer estrategias para la regulación del comportamiento de la población. Quintanilla (2004) plantea que la empatía permite que los agentes sean intérpretes del otro y comprendan sus creencias enfrentándose a su mundo de la vida; es desde allí que se tejen creencias comunes que pueden tener el agente y el intérprete, por lo cual refiere que el principio de caridad connota el compartir la creencia de cómo es el mundo pero también de cómo debería serlo. Por lo anterior, se afirma que la ética surge de la caridad desde un marco de solidaridad y tolerancia.

La importancia que le dan los cuidadores al cuidado desde una perspectiva ética de sensibilidad y compromiso, concuerda con los planteamientos de Boff (2002) para quien el cuidado en todas sus manifestaciones implica en primera instancia un sentido ético. Esta perspectiva también converge con los planteamientos de Nussbaum (2012) en cuanto a que el cuidado se relaciona con el amor, tanto con emociones como con las formas de comportarse de los sujetos mediadas por normas sociales; y con Gilligan (1982), en el sentido de establecer un diálogo en el que se visualice las particularidades de cada individuo, permitiendo que su sí mismo se construya.

Significa lo anterior que para los cuidadores su labor reviste un alto sentido ético manifiesto en la sensibilidad, el reconocimiento, el respeto, el compromiso y la responsabilidad, enmarcados en el amor y la comprensión hacía los niños, niñas, adolescentes y adultos que cuidan, pues para ellos estos componentes son importantes en la formación de vidas donde muchos de sus derechos fueron vulnerados; esto permite también identificar cómo el cuidado para los cuidadores de la institución se relaciona con un proceso de protección donde la población logre sentirse cuidada.

\section{- Perfil del cuidador}

Esta categoría emerge del análisis realizado con el propósito de hacer una identificación de las características del cuidador, partiendo no sólo de lo observado en las prácticas de cuidado sino haciendo explícita la percepción del cuidador sobre su labor.

De acuerdo con los cuidadores, existen unas características básicas que debe tener quien desarrolle las labores de atención y cuidado, adquiridas por su experiencia (figura 1), estas son: amor por lo que hace, tolerancia, vocación, empatía, liderazgo, respeto y capacidad de comprensión. Son características necesa- 
rias para desarrollar la labor de atención y cuidado, debido a la población que se atiende, para promover y fortalecer la capacidad de resiliencia tanto de los cuidadores como en la población.

“...el trabajo por hacerlo sin hacerlo con amor prácticamente como si ellos fueran nuestros hijos así digan que ellos no son nuestros hijos, entre comillas, pero es como si fueran nuestros hijos, a mí no me gustaría que mi hija andara por ahí toda "deschalengada" que yo le pagara a una señora para que me la cuidara bien y yo la viera toda sucia, mocosa, poposiada, orinada, no me gustaría, entonces como no me gustaría, tampoco se lo haría a ellos entonces yo a ellos les explico cómo deben ser las cosas" (Entrevista C6).

Por otra parte, algunos de los funcionarios de la institución son padres y madres de familia lo que implica que continúen ejerciendo un rol de cuidado en su hogar; también pueden tener dos trabajos o estudiar una carrera universitaria lo que reduce su vida social generando en algunas ocasiones molestias emocionales y físicas por una posible sobrecarga (Freudenberger y Richelson, 1980).

“...hay una sobrecarga emocional en este tipo de trabajo, una sobrecarga emocional que viene relacionada con el hecho de que nosotros permanecemos una gran cantidad de...de...de...del día de horas del día, acá dentro de la institución o dentro del centro ¿sí? Independientemente de todo nosotros también tenemos familia, pero en sí, la mayor parte del tiempo permanecemos acá y entonces primero existe una sobrecarga emocional” (Entrevista C8).

Las experiencias y prácticas diarias de cuidado les permiten configurarse como cuidador, lo cual está relacionado con lo expuesto por Heidegger (1951) quien menciona que en la acción de cuidado se configura el ser cuidador, por esto se hace importante fortalecer las prácticas de autocuidado ya que en ocasiones son pocas o nulas; por ello es que el cultivo de sí como lo menciona Michael Foucault (1987) configura el proceso de visualización para el cuidado propio desde un ejercicio de libertad.

Los resultados concuerdan con los hallazgos de investigaciones publicadas por Albert Gallart (2004), Villarejo et al. (2012) en términos de las consecuencias en la salud física, emocional y mental de los cuidadores que implica realizar la labor de cuidado de manera satisfactoria, pues se desarrollan molestias progresivas que pueden llevar a que en el cuidador se produzca la percepción de sobrecarga laboral, repercutiendo en el desarrollo del síndrome de burnout (Hernández y Ehrenzweigs, 2008). Sin embargo, no se encuentran resultados que evidencien la percepción del cuidado en cuidadores de niños, niñas, adolescentes y adultos con trastorno de la conducta o trastorno mental psicosocial que estén institucionalizados.

Para los cuidadores es importante tener conocimientos sobre las problemáticas de la población o procesos de atención, ya que muchos se encargan de los medicamentos, alimentación o primeros auxilios; además, deben saber manejar episodios que se dan en los beneficiarios debido a su condición mental, neurológica o física, por lo tanto, se puede afirmar que el cuidado no sólo es asistencial sino que también comprende unos conocimientos especializados que no son de orden técnico, de ahí que se deba tener un equipo interdisciplinario para la respuesta de necesidades de la población.

“...Teniendo en cuenta, primero, que tiene un diagnóstico de base y, segundo, que está bajo una medicación, que es una medicación psiquiátrica, ¿sí? y que a su vez esa medicación psiquiátrica tiene como efectos secundarios, un deterioro en sus procesos cognitivos. Entonces yo tengo también que acomodar mi lenguaje en ese sentido de... de...de cómo yo me voy a dirigir a las personas, de cómo yo me voy a hacer entender, de cómo yo voy incluso a dar 
una instrucción de forma tal que pueda ser comprensible para esa persona y que la pueda ejecutar de una manera adecuada, ahí se vería la parte de los conocimientos técnicos. ¿Qué sucede? Esos son los conocimientos que de pronto desde esa parte de la disciplina se conoce, pero... bueno hay muchos cuidadores o formadores que llegan quizá en blanco ¿sí? Hay muchas personas que quizá, por ejemplo en el caso de los auxiliares de enfermería como tal, eh tienen digamos una experiencia relativa con algún...en algún trabajo, con algún tipo de población similar pero en población digamos directamente como la que nosotros tenemos, no la poseen ¿sí? Entonces ¿qué hay que hacer?...comenzar a formar desde ahí, y comenzar a enseñar, comenzar a orientar, comenzar digamos a capacitar en cierta forma, de cómo va hacer el trabajo con la población que se está....que se atiende acá..." (Entrevista C8).

\section{Conclusiones}

La investigación realizada permitió avanzar en contenido que evidencie la percepción del cuidador frente a su labor, pues como se indicó en algunos apartados de este artículo, no se evidencian procesos de investigación en los que se interprete las significaciones de cuidado en cuidadores de niños, niñas, adolescentes y adultos que se encuentran institucionalizados y presentan algún trastorno de la conducta o trastorno psicosocial.

Los resultados permitieron interpretar, comprender y confirmar que el cuidado trasciende la labor médica o de enfermería, pues todos los funcionarios de la institución están involucrados en una labor de cuidado que busca la formación de la población orientada al restablecimiento de los derechos que en su momento fueron vulnerados, siendo percibido en los funcionarios como una actitud, más que como un simple cumplimiento del deber; no obstante, también afirman la importancia de conocimientos técnicos para el cuidado dado el perfil de población que cuidan.

Al realizar la descripción de las prácticas de cuidado de los cuidadores de la institución, se observó que en los diferentes espacios en los que los cuidadores efectúan las labores, se otorga un alto significado a estas, manifestado en el interés y la relevancia que le dan al conocimiento de las problemáticas e historia de vida de cada uno de los niños, niñas, adolescentes y adultos atendidos; viéndolos como personas a quienes se les debe responder por sus necesidades particulares con la dedicación y el compromiso que se requiere, siendo el amor y la comprensión los pilares fundamentales para desarrollar su práctica, comprendiendo de esta manera que el cuidado está enmarcado en estos componentes.

No obstante, la labor de cuidado está asociada a una actividad que presenta dificultades debido a las tensiones que emanan entre lo que demanda la institución operadora encargada de la atención de la población y la entidad del Estado contratante, en relación a las prácticas que los funcionarios desarrollan. Es decir, el cuidador lleva a cabo su trabajo realizando distintas actividades pero refieren que están en constante supervisión, lo que influye en el desempeño de su trabajo.

Por otra parte, desde las prácticas de cuidado y la relación entre cuidadores y los beneficiarios, se permitió reconocer el mundo que intersubjetivamente han construido. Sus aportes contribuyen a repensar el concepto de cuidado como una manera de proteger la vida y garantizar condiciones óptimas para el desarrollo humano de quién es cuidado y del cuidador. 
Al describir e interpretar las prácticas de atención y de cuidado se comprende que es una labor enmarcada en la paciencia, amor, vocación y comprensión sobre las necesidades de quien se cuida, con tensiones de diferente tipo, es decir, que el ejercicio de cuidado requiere de un tipo de persona específico que, como lo nombran los cuidadores, necesita un espíritu que enfrente las adversidades en pro del bienestar (Mulsow, 2008) de quién es cuidado y de sí mismo.

Es de mencionar que, si bien los cuidadores presentan conocimientos técnicos con los cuales diseñan estrategias de intervención con la población que atienden, sin embargo, al manifestarse la particularidad de cada uno de ellos como sujeto, sumado a las situaciones emergentes que puedan surgir por sus alteraciones comportamentales, se presentan momentos en los que el cuidador debe planear alternativas de solución que son aprendidas desde la misma práctica y el caso particular que aborde, lo que conlleva concluir que el cuidado no es estático y que ello conlleva también que se presenten tensiones en las que el cuidador pueda ejercer una práctica de cuidado autoritaria, la cual se contrasta con los componentes de amor, comprensión y dedicación que para ellos se enmarcan en su labor.

A partir de los resultados obtenidos en la investigación se evidencia que, producto de las tensiones y dificultades, existe en los funcionarios un cansancio manifiesto; esto es debido al horario de 14 horas que deben dedicar a la labor, a la tensión en la relación entre compañeros que en ocasiones se presenta, así como a las alteraciones comportamentales que constantemente se presentan en la población, lo que conlleva al agotamiento. Frente a esta situación se concluye que es importante que las instituciones formulen acciones orientadas al cuidado del cuidador con el fin de prevenir la deserción laboral, problemas interpersonales o posibles maltratos a la población; de igual manera, es necesario trabajar en estrategias de afrontamiento y en mecanismos de solución de problemas que promuevan el reconocimiento mutuo, el respeto y la tolerancia. En otros términos, es indispensable fortalecer la ética del cuidado entre los miembros de la comunidad institucional.

Es por todo lo anterior que surge la necesidad de dar continuidad a estudios que permitan desde el ejercicio de la interpretación ahondar en las posturas, experiencias, opiniones y percepciones desde los discursos de quienes realizan esta labor día a día, los cuales contribuyen a entender mejor la misma. Además, es importante fortalecer el reconocimiento de las habilidades que las personas cuidadas pueden desempeñar en espacios sociales, pues esto permitiría espacios en los que el cuidador realice actividades para su cuidado propio, ya que su labor sería apoyada por instituciones en las que vincularían escolar o laboralmente a la persona que cuida previniendo que personas no sólo con los diagnósticos abordados en esta investigación sino demás tipologías mentales o de comportamiento no sean maltratadas debido a la sobrecarga que pueda sentir su cuidador, bien sea informal o formal.

Finalmente, desde las percepciones de cuidado de los cuidadores, emerge el cuestionamiento sobre la formación profesional en este campo en Colombia, ya que muchos de los cuidadores desarrollan sus actividades a partir del aprendizaje adquirido por la experiencia, pero ninguno de los cuidadores del estudio recibió educación especializada en aspectos básicos como el cuidado del otro y el cuidado de sí, enmarcado en las prácticas y relaciones de cuidado. 


\section{Referencias bibliográficas}

Aguas, S. (1999): “Una protección social invisible”. Hechos y Derechos, 6: 27-37.

American Psychiatric Association (2014a): DSM-5. Manual diagnóstico y estadístico de los trastornos mentales. Arlington: American Psychiatric Association.

American Psychiatric Association (2014b): Guía de consulta de los criterios diagnósticos del DSM- 5. Arlington: American Psychiatric Association.

Báez-Hernández, F. J. et al. (2009): "El significado de cuidado en la práctica profesional de enfermería”. Revista Aquichan, 9 (2): 127-134

Berlin, L. J. (2005): "Interventions to enhance early attachments. The state of the field today", en Berlin, L.J. et al. (eds.): Enhancing early attachments: Theory, research, intervention, and policy. New York: The Guilford Press.

Berlin, L. J, et al. (2008): "Prevention and intervention programs for supporting early attachment security", en Cassidy, J. y Shaver, P. R. (eds.): Handbook of attachment: Theory, research, and clinical application. Nueva York: The Guilford Press.

Boff, L. (2002): El cuidado esencial: ética de lo humano, compasión por la tierra. Madrid: Trotta.

Carretero, S. et al. (2006): La sobrecarga de las cuidadoras de personas dependientes, análisis y propuestas de intervención psicosocial. Valencia: Tirant lo Blanch.

Cerquera, A. M. y Galvis, M. J. (2014): "Efectos de cuidar personas con Alzheimer: un estudio sobre cuidadores formales e informales". Pensamiento Psicológico, 12 (1), 149-167.

Corte Constitucional (2015): Constitución Política de Colombia. Actualizada con los Actos Legislativos a 2015. Bogotá, Colombia: Imprenta Nacional.

De la Cuesta, C. (2004): Cuidado Artesanal: la invención ante la adversidad. Antioquía, Colombia: Universidad de Antioquía.

De los Reyes, M. C. (2001): "Construyendo el concepto cuidador de ancianos", en: IV Reunión De Antropología Do Mercosul. Foro de Investigación: Envejecimiento de la población en el Mercosur. Curitiba, Brasil.

Espinoza, M. et al. (2011): "Vivencia holística del estrés en profesionales de Enfermería". Revista Enfermería Actual de Costa Rica, 20

Flores J. A. et al. (1997): "Psicopatología de los cuidadores habituales de ancianos”. Jano, 3 (1218): 261-272.

Foucault, M. (1987): Historia de la sexualidad 3: la inquietud de sí. Madrid: Siglo XXI.

Freudenberger, H. J. y Richelson, G. (1980): Burnout: The high cost of high achievement. New York: Anchor Press.

Gallart, A. (2004): "Factores de riesgo del cansancio en el cuidador formal no profesional inmigrante en Barcelona". Revista de Administración Sanitaria Siglo XXI, 2 (2): 299-316.

Gilligan, C. (1982): La moral y la Teoría: Psicología del desarrollo femenino. México D.F.: Fondo de Cultura Económica.

Granadillo, M. (2010): Herramientas a padres y docentes para atender a niños con diagnóstico de TDAH con síntomas de TOD. Proyecto de especialización. Medellín: Universidad CES. 
Gutiérrez Aceves, G. A. et al. (2006): "Síndrome de Burnout”. Medigraphic. 11 (4): 305-309.

Harris, D. y Guten, S. (1979): "Health-protective behavior: an exploratory study". Journal of Health and Social Behavior. 20 (1): 17-29.

Heidegger, M. (1951): El Ser y el Tiempo. México: Fondo de Cultura Económica.

Hernández, Z. E. y Ehrenzweig, Y. (2008): "Percepción de sobrecarga y nivel de burnout en cuidadores formales del adulto mayor institucionalizado". Enseñanza e investigación en psicología, 1 (13): 127-142.

Instituto Colombiano de Bienestar Familiar (ICBF) (2006): Lineamiento técnico para la atención de niños, niñas, adolescentes y mayores de 18 años con derechos inobservados, amenazados o vulnerados, con discapaci-

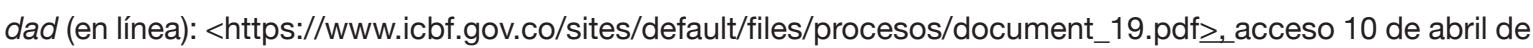
2017.

Kohlberg, L. (1981): Philosophy of Moral Development: Moral Stages and the Idea of Justice. San Francisco: Harp Row Publishers.

Luckmann, T. (2008): “Conocimiento, acción e interpretación”, en Luckmann, T.: Conocimiento y Sociedad. Madrid: Trotta.

Meana, M. Á. (2009): Síndrome de burnout: su prevalencia en una población de médicos en programas intensivos de capacitación en la especialidad de Clínica Médica. Trabajo de especialización. Buenos Aires: Universidad Nacional de la Plata.

Milosavljevic, V. (2007): Estadísticas para la equidad de género: magnitudes y tendencias en América Latina. Nueva York: Naciones Unidas.

Mulsow, G. (2008): “Desarrollo emocional: impacto en el desarrollo humano”. Educação, 1 (31): 61-65.

Murueta, E. M. (2009): “Cura y Praxis”, en Guerra, R. \& Yáñez, A. (coords.): Martín Heidegger. Caminos. Cuernavaca: Universidad Nacional Autónoma de México.

Noddings, N. (2005): Caring in education (en línea): <http://infed.org/mobi/caring-in-education/>, acceso 5 de mayo de 2016.

Nussbaum, M. (2012): Las mujeres en el desarrollo humano. Barcelona: Herder.

Organización Mundial de la Salud, OMS (2004): Prevención de los Trastornos Mentales-Intervenciones Efectivas y Opciones de Políticas, Informe Compendiado. Ginebra: Organización Mundial de la Salud.

Organización Mundial de la Salud (2001): Clasificación Internacional de Enfermedades (CIE-10). Ginebra: Organización Mundial de la Salud.

Pourtois, J. P. y Desmet, H. (1992): Epistemología e Instrumentación en Ciencias Humanas. Barcelona: Herder.

Quintanilla, P. (2004): “Comprender al otro es crear un espacio compartido: caridad, empatía y triangulación”. Revista Ideas y Valores, 125 (53): 81-97.

Santamaría, C. (2013): Caracterización del estilo de crianza de los niños con trastornos de comportamiento disocial y negativista desafiante del centro de protección Jacinta y Francisco. Tesis doctoral. Madrid: Universidad Autónoma de Madrid.

Secretaría de Integración Social y Universidad Nacional de Colombia (2008): Elementos de una guía para cuidadores de personas en situación de discapacidad. Bogotá: Departamento de Ocupación Humana. 
Stake, E. R. (1998): Investigación con estudio de casos. Madrid: Morata.

Torres, J. (2010): Aspectos psicológicos en cuidadores formales de ancianos: carga y afrontamiento del estrés: un estudio en población socio sanitaria. Huelva: Universidad de Huelva.

UNICEF (2006): Excluidos e invisibles: Estado Mundial de la Infancia. Nueva York: UNICEF.

Valderrama H. (1999): "La institución geriátrica como centro de servicios gerontológicos". En Primer Congreso sobre organizaciones geriátricas. Panel llevado a cabo en el congreso de la Federación de Geriátricos de la República Argentina.

Valles, M. (1999): Técnicas cualitativas de Investigación Social. Madrid: Editorial Síntesis, S.A.

Villarejo, L. et al. (2012): "Sobrecarga y dolor percibido en cuidadoras". Revista electrónica trimestral de enfermería, 27: 159-165.

Watson, J. (1979): Nursing: The philosophy and science of caring. Boston: Little Brown.

Yin, R. (1984): Case Study Research. Desing and Methods. London: Sage Publications.

Zingraff, M. et al. (1993): “Child maltreatment and youthful problem behavior”. Criminology, 2 (31): 173-202. 